\title{
Constraining top-Higgs couplings at high and low energy
}

\author{
Emanuele Mereghetti ${ }^{1, a}$ \\ ${ }^{1}$ Theoretical Division, Los Alamos National Laboratory, Los Alamos, NM 87545, USA
}

\begin{abstract}
The study of the couplings of the Higgs boson and of the top quark plays a preeminent role at the LHC, and could unveil the first signs of new physics. I will discuss the interplay of direct and indirect probes of certain classes of top and Higgs couplings. Including constraints from collider observables, precision electroweak tests, flavor physics, and electric dipole moments (EDMs), I will show that indirect probes are competitive, if not dominant, for both the CP-even and CP-odd top and Higgs couplings we considered. I will discuss the role of theoretical uncertainties, associated with hadronic and nuclear matrix elements, and indicate targets to further improve the constraining power of EDM experiments.
\end{abstract}

\section{Introduction}

The Standard Model (SM) of particle physics is a successful theory, that has been validated over a large range of energy scales. Its latest success is the discovery of the Higgs particle at the Large Hadron Collider (LHC) [1, 2], whose properties are in agreement with SM expectations [3], within the still sizable experimental uncertainties. On the other hand, we have several hints of physics beyond the SM (BSM), and the SM could just be the low-energy effective field theory of a more complete theory. If the scale of BSM physics is much larger than the electroweak scale, new physics might primarily manifest in deviations of the properties of SM particles from the SM expectations. In this talk I will focus on the Higgs boson and on the top quark. I will introduce a class of operators that modifies the Higgs and top interactions, and I will study the constraints on the real and imaginary part of their couplings, at high and low energy. This talk is based on Refs. [4-6]

\section{The SM EFT}

In this work, we describe new physics in the framework of the SM Effective Field Theory (EFT) [7-11]. The main assumptions of this framework are that the scale of new physics $\Lambda$ is much larger than the electroweak (EW) scale, and that there are no new light degrees of freedom beyond SM fields. Under these assumptions, new physics is parameterized by a complete set of operators, invariant under the SM gauge group $S U(3)_{c} \times S U(2)_{L} \times U(1)_{Y}$, and of canonical dimension larger than four. Our last assumption is that the importance of the higher-dimensional operators is determined by their canonical dimension, so that the most relevant operators have dimension six. The full set of dimension-six operators has been known for some time [7,8]. Here we focus on a subset, namely those operators

ae-mail: emereghetti@lanl.gov 
that contain interactions of the top quark and the Higgs boson, and flip the chirality of the top. At dimension six, there are five such interactions: a non-standard Yukawa coupling, one hypercharge dipole, one gluonic dipole, and two electroweak dipoles. Working in the unitary gauge, in which the Higgs doublet takes the form $\varphi=(0, v+h)^{T} / \sqrt{2}$, and neglecting flavor changing neutral currents, the effective Lagrangian at the new physics scale $\Lambda \gg v$ is

$$
\mathcal{L}_{\text {top }}=\sum_{\alpha \in\{Y, g, \gamma, W t, W b\}} C_{\alpha} O_{\alpha}+\text { h.c. }
$$

with complex couplings $C_{\alpha}=c_{\alpha}+i \tilde{c}_{\alpha}$ and

$$
\begin{aligned}
O_{Y} & =-m_{t} \bar{t}_{L} t_{R}\left(v h+\frac{3}{2} h^{2}+\frac{1}{2} \frac{h^{3}}{v}\right), \quad O_{\gamma}=-\frac{e Q_{t}}{2} m_{t} \bar{t}_{L} \sigma_{\mu \nu}\left(F^{\mu v}-t_{W} Z^{\mu v}\right) t_{R}\left(1+\frac{h}{v}\right), \\
O_{g} & =-\frac{g_{s}}{2} m_{t} \bar{t}_{L} \sigma_{\mu \nu} G^{\mu v} t_{R}\left(1+\frac{h}{v}\right), \\
O_{W t} & =-g m_{t}\left[\frac{1}{\sqrt{2}} \bar{b}_{L}^{\prime} \sigma^{\mu v} t_{R} W_{\mu v}^{-}+\bar{t}_{L} \sigma^{\mu v} t_{R}\left(\frac{1}{2 c_{W}} Z_{\mu v}+i g W_{\mu}^{-} W_{v}^{+}\right)\right]\left(1+\frac{h}{v}\right), \\
O_{W b} & =-g m_{b}\left[\frac{1}{\sqrt{2}} \bar{t}_{L}^{\prime} \sigma^{\mu v} b_{R} W_{\mu \nu}^{+}-\bar{b}_{L} \sigma^{\mu v} b_{R}\left(\frac{1}{2 c_{W}} Z_{\mu v}+i g W_{\mu}^{-} W_{v}^{+}\right)\right]\left(1+\frac{h}{v}\right),
\end{aligned}
$$

where $Q_{t}=2 / 3, t_{W}=\tan \theta_{W}, c_{W}=\cos \theta_{W}$, with $\theta_{W}$ the Weinberg angle. The operators $O_{W t, W b}$ contain the combinations $b^{\prime}=V_{t b} b+V_{t s} s+V_{t d} d$, and $t^{\prime}=V_{t b}^{*} t+V_{c b}^{*} c+V_{u b}^{*} u$, where $V_{i j}$ are CKM matrix elements. The couplings $C_{\alpha}$ have mass dimension -2, and we will give bounds on the dimensionless combination $v^{2} C_{\alpha} \sim v^{2} / \Lambda^{2}$.

In this work we assume that new physics is only present in the five operators in Eq. (1), that is, we assume that at the scale $\Lambda$ only the five complex coefficients in Eq. (1) are non-vanishing. We consider how the operators in Eq. (1) affect collider observables involving the Higgs and top quark, including Higgs production via gluon fusion, Higgs production in association with a $t \bar{t}$ pair, and single top production. We then integrate out heavy SM degrees of freedom, and consider how anomalous top couplings affect low energy observables, in particular rare $B$ meson decays and the neutron and electron electric dipole moments (EDMs).

\section{Collider constraints}

The modifications of the top and Higgs couplings induced by the operators in Eq. (1) can manifest themselves in collider observables in two ways. First of all, these operators affect processes in which the top quark is directly produced, possibly in association with the Higgs or with electroweak bosons. For example, a non-vanishing $C_{g}$ modifies the $t \bar{t}$ and $t \bar{t} h$ production cross sections, while $C_{W t}$ and $C_{W b}$ correct single top production. Secondly, these operators can be probed indirectly, by studying processes that have no real top in the final state, but are sensitive to loops with virtual tops. Two examples are the gluon fusion Higgs production cross section, $g g \rightarrow h$, and the Higgs decay into two photons, $h \rightarrow \gamma \gamma$. These processes are generated at one loop in the SM, and thus are particularly sensitive to modifications of the top couplings. We will see that the best bounds on the real part of $C_{Y}$, $C_{g}$ and $C_{\gamma}$ come indeed from the Higgs production cross section and branching ratios.

The direct observables we considered in our analysis are $t \bar{t}, t \bar{t} h$ and $t$-channel single top production. For $t \bar{t}$ and $t \bar{t} h$, we define the signal strength as

$$
\mu_{t \bar{t}}=\frac{\sigma_{p p(\bar{p}) \rightarrow t \bar{t}}}{\sigma_{p p(\bar{p}) \rightarrow t \bar{t}}^{S M}}, \quad \mu_{t \bar{t} h}=\frac{\sigma_{p p \rightarrow t \bar{t} h}}{\sigma_{p p \rightarrow t \bar{t} h}^{S M}} .
$$


The cross section induced by the top chromo-magnetic (CMDM) and chromo-electric (CEDM) dipole moments, $c_{g}$ and $\tilde{c}_{g}$, was first computed in Refs. [12,13]. Using the results of Ref. [6] we find

$$
\begin{aligned}
\mu_{t \bar{t}}(1.96 \mathrm{TeV}) & =\left(1-(1.4 \pm 0.1)\left(v^{2} c_{g}\right)+(0.95 \pm 0.11)\left(v^{2} c_{g}\right)^{2}+(0.33 \pm 0.07)\left(v^{2} \tilde{c}_{g}\right)^{2}\right) \\
\mu_{t \bar{t}}(8 \mathrm{TeV}) & =\left(1-(1.3 \pm 0.1)\left(v^{2} c_{g}\right)+(1.9 \pm 0.2)\left(v^{2} c_{g}\right)^{2}+(1.3 \pm 0.2)\left(v^{2} \tilde{c}_{g}\right)^{2}\right),
\end{aligned}
$$

with $\sigma_{p \bar{p} \rightarrow t \bar{t}}^{S M}(1.96 \mathrm{TeV})=7.45 \pm 0.44 \mathrm{pb}$ and $\sigma_{p p \rightarrow t \bar{t}}^{S M}(8 \mathrm{TeV})=252.9 \pm 20 \mathrm{pb}$, at the factorization and renormalization scale $\mu=m_{t}$. The SM $t \bar{t}$ cross section has been computed using the program TOP ++ [14], and it includes next-to-next-to-leading order $\left(\mathrm{N}^{2} \mathrm{LO}\right)$ corrections [15]. The contribution of $C_{g}$ is only included at LO. The theoretical errors are determined by PDF and scale variations.

The top CMDM and CEDM also modify the $t \bar{t} h$ cross section, which is also affected by the Yukawa coupling $C_{Y}$. At $\sqrt{S}=8 \mathrm{TeV}$ we find

$$
\begin{aligned}
\mu_{t \bar{t} h}(8 \mathrm{TeV})= & \left(1+v^{2} c_{Y}\right)^{2}+(0.33 \pm 0.02)\left(v^{2} \tilde{c}_{Y}\right)^{2}-(7.11 \pm 0.02)\left(v^{2} c_{g}\right)+(52 \pm 5)\left(v^{2} c_{g}\right)^{2} \\
& +(44 \pm 4)\left(v^{2} \tilde{c_{g}}\right)^{2}-(11.0 \pm 0.1)\left(v^{2} c_{g}\right)\left(v^{2} c_{Y}\right)-(0.12 \pm 0.16)\left(v^{2} \tilde{c}_{g}\right)\left(v^{2} \tilde{c}_{Y}\right) .
\end{aligned}
$$

The SM $t \bar{t} h$ cross section at $8 \mathrm{TeV}$ is $\sigma_{p p \rightarrow t \bar{h}}^{S M} \sim 0.128 \mathrm{pb}$ [16]. From Eqs. (4) and (5), we see that, in absolute terms, the contribution of $C_{g}$ to $t \bar{t}$ is larger than to $t \bar{t} h$. The relative correction to $t \bar{t} h$, however, is a factor of five larger than the correction to $t \bar{t}$. Thus, for equal experimental and theoretical uncertainties, the associated production of a $t \bar{t}$ pair and a Higgs would be more sensitive to the top CMDM than $t \bar{t}$. With current experimental input from the Tevatron and LHC experiments, the $t \bar{t}$ cross section is known with roughly $5 \%$ uncertainty, while the $t \bar{t} h$ signal strength has large $O(1)$ errors $[17,18]$. Thus, at the moment, $t \bar{t}$ gives a stronger bounds on $C_{g}$, but data from the LHC Run II could greatly improve the bounds from $t \bar{t} h$. In our analysis, we include data on $t \bar{t}$ production from the Tevatron experiments [19] at $\sqrt{S}=1.96 \mathrm{TeV}$, and from the ATLAS and CMS experiments at $\sqrt{S}=8 \mathrm{TeV}[20,21]$. For $t \bar{t} h$, we used the signal strengths as measured by the ATLAS and CMS collaborations $[3,17,18]$.

Direct constraints on $C_{\gamma}$ could be obtained by studying the associated production of a $t \bar{t}$ pair and a photon or $Z$ boson [22, 23]. Since the ensuing bounds are relatively weak, we did not include these processes in our analysis.

Finally, $C_{W t}$ and $C_{W b}$ affect single top production, and the decay of the top into a $W$ and a $b$ quark. The contribution of $C_{W b}$ is suppressed by the bottom Yukawa, and it is negligible. At NLO in QCD, the inclusive $t$-channel single top cross section in the presence of the operator $C_{W t}$ is

$$
\begin{aligned}
& \frac{\sigma_{t}(8 \mathrm{TeV})}{\mathrm{pb}}=(56.4 \pm 2.4)-(11.7 \pm 0.3) v^{2} c_{W t}+(21.0 \pm 0.5)\left(\left(v^{2} c_{W t}\right)^{2}+\left(v^{2} \tilde{c}_{W t}\right)^{2}\right), \\
& \frac{\sigma_{\bar{t}}(8 \mathrm{TeV})}{\mathrm{pb}}=(30.7 \pm 1.3)-(0.5 \pm 0.2) v^{2} c_{W t}+(7.7 \pm 0.2)\left(\left(v^{2} c_{W t}\right)^{2}+\left(v^{2} \tilde{c}_{W t}\right)^{2}\right) .
\end{aligned}
$$

The cross sections were obtained by setting the factorization and renormalization scales to $\mu=m_{t}$, where we also evaluate the couplings $c_{W t}$ and $\tilde{c}_{W t}$. The errors take into account scale and PDF variations.

The angular distributions of the charged lepton in the leptonic decay of the $W$ boson produced in top decays provide strong constraints on the $t W b$ coupling, and its helicity structure. The lepton momentum is determined by the polar and azimuthal angles $\theta$ and $\phi$, defined in the $W$ rest frame, with the $z$ axis oriented in the direction of the $W$ momentum in the lab frame and the $y$ axis orthogonal to $W$ and jet momenta. The measurement of $\theta$ determines the three helicity fractions, $F_{0}$, the fraction of 


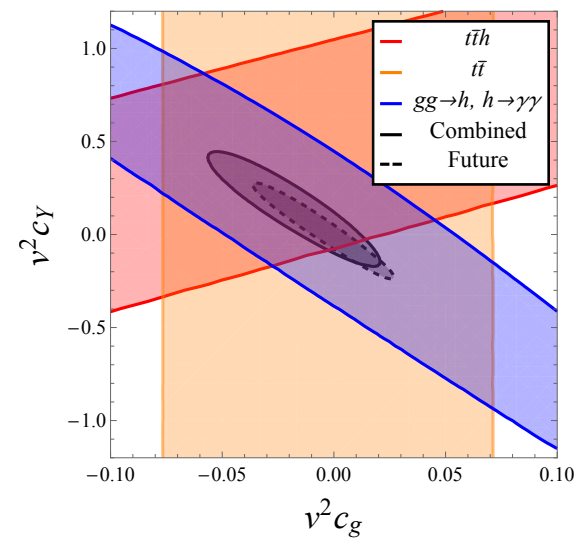

Figure 1. 90\% C.L. allowed regions in the $v^{2} c_{g}-v^{2} c_{Y}$ plane, with couplings evaluated at $\Lambda=1 \mathrm{TeV}$. We assume that only $C_{g}$ and $C_{Y}$ are generated at the high scale and the theoretical uncertainties are dealt with using the R-fit procedure.

longitudinally-polarized $\mathrm{W}$ bosons, and $F_{L, R}$, the fractions of left/right-handed transversely-polarized $\mathrm{W}$ bosons. In addition, the $\phi$ distribution is sensitive to the phase of the weak dipole $C_{W t}[24,25]$. The SM contribution to the helicity fractions is known at $\mathrm{N}^{2} \mathrm{LO}$ in QCD [26], while the corrections induced by the operators $C_{W t}$ and $C_{W b}$ are known at NLO in QCD [27]. As for single top, $C_{W b}$ is suppressed by the bottom Yukawa, resulting in weak bounds.

The loop processes that are most sensitive to anomalous top couplings are $g g \rightarrow h$ and $h \rightarrow \gamma \gamma$. The corrections to these processes can be obtained by considering threshold corrections and mixing of the operators in Eq. (1) onto the operators $g_{s}^{2} C_{\varphi G} \varphi^{\dagger} \varphi G_{\mu \nu} G^{\mu \nu}$ and $-g_{s}^{2} C_{\varphi \tilde{G}} \varphi^{\dagger} \varphi G_{\mu \nu} \tilde{G}^{\mu \nu}$, where $\tilde{G}_{\mu \nu}=$ $\varepsilon_{\mu \nu \alpha \beta} G^{\alpha \beta} / 2$, and similar operators with EW bosons. The anomalous scalar and pseudoscalar Yukawa $c_{Y}$ and $\tilde{c}_{Y}$ contribute to $C_{\varphi G}$ and $C_{\varphi \tilde{G}}$ at the top threshold, in the same way as the SM Yukawa. The color dipoles mix with $C_{\varphi G}$ and $C_{\varphi \tilde{G}}$, and, similarly, the electromagnetic dipoles mix with $\varphi^{\dagger} \varphi F_{\mu \nu} F^{\mu \nu}$ and $\varphi^{\dagger} \varphi F_{\mu \nu} \tilde{F}^{\mu \nu}$. Solving the RGE, we get that the production and decay signal strengths are [6]

$$
\begin{aligned}
\frac{\sigma_{g g F}}{\sigma_{g g F}^{S M}} & =\left(1+v^{2} c_{Y}\left(m_{t}\right)+7.6 v^{2} c_{g}(\Lambda)\right)^{2}+\left(1.5 v^{2} \tilde{c}_{Y}\left(m_{t}\right)+7.6 v^{2} \tilde{c}_{\gamma}(\Lambda)\right)^{2} \\
\frac{\Gamma_{h \rightarrow \gamma \gamma}}{\Gamma_{h \rightarrow \gamma \gamma}^{S M}} & =\left(1-0.3 v^{2} c_{Y}\left(m_{t}\right)+2.0 v^{2} c_{\gamma}(\Lambda)\right)^{2}+\left(0.4 v^{2} \tilde{c}_{Y}\left(m_{t}\right)-2.0 v^{2} \tilde{c}_{\gamma}(\Lambda)\right)^{2}
\end{aligned}
$$

The remaining operators, $C_{W t}$ and $C_{W b}$, do not significantly affect these processes. Eq. (7) shows that $c_{g}$ gives a large correction to $g g \rightarrow h$. Since the signal strength is known with $10 \%$ uncertainties [3], the bound on $c_{g}$ is strong, a few percent in a single coupling analysis. In the $\mathrm{SM} h \rightarrow \gamma \gamma$ is dominated by $W$ loops, which somewhat dilute the impact of $c_{\gamma}$ and $c_{Y}$. Also in this case, the signal strength is known with roughly $10 \%$ accuracy, leading to a 10\% limit on $c_{\gamma}$ (and a weaker bound on $c_{Y}$ ).

In Fig. 1 we show the $90 \%$ confidence level (C.L.) collider bounds on $c_{Y}$ and $c_{g}$ from direct and indirect probes. The black region shows the combined bound, and the dashed black contour denotes a projection of future LHC bounds, assuming a measurement of the $t \bar{t} h$ signal strength with $30 \%$ uncertainties $[28,29]$. The present data still allow for large deviations from the SM Yukawa coupling, up to $50 \%$ if one allows for both $c_{g}$ and $c_{Y}$ to be non-zero at the scale $\Lambda$. Future, more precise measurements of $t \bar{t} h$ are crucial to improve this constraint. 


\section{Constraints from Flavor Physics and Electric Dipole Moments}

Next we study the impact of top anomalous couplings on low energy processes, including $B \rightarrow X_{s} \gamma$, the muon $g-2$, and the electron and neutron EDMs. For $B \rightarrow X_{s} \gamma$, the experimental world average for the branching ratio and the $\mathrm{CP}$ asymmetry are [30, 31]

$$
\mathrm{BR}\left(B \rightarrow X_{s} \gamma\right)=(3.43 \pm 0.21 \pm 0.07) \times 10^{-4}, \quad A_{C P}\left(B \rightarrow X_{s} \gamma\right)=0.015 \pm 0.02 .
$$

As far as EDMs are concerned, the most sensitive probes are the EDMs of the electron, neutron and ${ }^{199} \mathrm{Hg}$. The $90 \%$ C.L. bounds on these EDMs are [32-35]

$$
\left|d_{e}\right|<8.7 \times 10^{-16} e \mathrm{fm}, \quad\left|d_{n}\right|<3.0 \times 10^{-13} e \mathrm{fm}, \quad\left|d_{H g}\right|<6.2 \times 10^{-17} e \mathrm{fm} .
$$

The muon $g-2$ is also precisely measured [30], and shows some tension with the SM expectation.

The top operators in Eq. (1) affect EDMs and $B \rightarrow X_{s} \gamma$ at the loop level, via threshold corrections and mixing into a different set of effective operators. The most important of these effective operators are

$$
\begin{aligned}
\mathcal{L}_{\text {low }}= & -\sum_{l=e, \mu} \frac{e Q_{e} m_{l}}{2} C_{\gamma}^{(l)} \bar{l}_{L} \sigma^{\mu v} l_{R} F_{\mu \nu}-\sum_{q=u, d, s} \frac{e Q_{q} m_{q}}{2} C_{\gamma}^{(q)} \bar{q}_{L} \sigma^{\mu v} q_{R} F_{\mu \nu}-\frac{e Q_{b} m_{b}}{2} C_{\gamma}^{(b s)} \bar{s}_{L} \sigma^{\mu v} b_{R} F_{\mu v} \\
& -\sum_{q=u, d, s} \frac{g_{s} m_{q}}{2} C_{g}^{(q)} \bar{q}_{L} \sigma^{\mu v} G_{\mu \nu} q_{R}-\frac{g_{s} m_{b}}{2} C_{g}^{(b s)} \bar{s}_{L} \sigma_{\mu \nu} G^{\mu v} b_{R}+\frac{g_{s} C_{\tilde{G}}}{3} f_{a b c} \tilde{G}_{\mu \nu}^{a} G_{\mu \rho}^{b} G_{v}^{c \rho}+\text { h.c. }
\end{aligned}
$$

The $B \rightarrow X_{s} \gamma$ branching ratio in terms of the flavor-changing dipoles $C_{\gamma}^{(b s)}$ and $C_{g}^{(b s)}$ is [36, 37]

$$
\begin{aligned}
B R\left(B \rightarrow X_{s} \gamma\right)= & (3.36 \pm 0.23) \times 10^{-4}\left\{1+7.9 \cdot 10^{2}\left(v^{2} c_{\gamma}^{(b s)}\right)+0.7 \cdot 10^{2}\left(v^{2} \tilde{c}_{\gamma}^{(b s)}\right)\right. \\
& \left.-9.8 \cdot 10^{2}\left(v^{2} c_{g}^{(b s)}\right)-1.8 \cdot 10^{2}\left(v^{2} \tilde{c}_{g}^{b s}\right)+\ldots\right\},
\end{aligned}
$$

where we used the SM prediction of Refs. [38-40], and ... include contributions that are quadratic in $c_{\gamma, g}^{(b s)}$. The coefficients in Eq. (12) are evaluated at the scale $\mu=m_{t}$. The CP asymmetry $A_{C P}$ has a more complicated expression, that can be found in Ref. [41], and it is affected by larger nonperturbative errors.

The electron EDM depends only on the imaginary part of the operator $C_{\gamma}^{(e)}$ in Eq. (11).

$$
d_{e}=e Q_{e} m_{e} \tilde{c}_{\gamma}^{(e)}\left(\Lambda_{\chi}\right)=-1.7 \times 10^{-9} v^{2} \tilde{c}_{\gamma}^{(e)}\left(\Lambda_{\chi}\right) e \mathrm{fm},
$$

where $\Lambda_{\chi}$ is a scale of order $1 \mathrm{GeV}$. The neutron EDM depends on the remaining flavor diagonal operators in Eq. (11). In this case, expressing the EDM in terms of the quark-gluon operators in Eq. (11) requires the calculation of hadronic matrix elements, which are at the moment poorly known. Combining information from QCD sum rules [42-46], lattice QCD calculations [47, 48] and naive dimensional analysis $[49,50]$, we can write

$$
\begin{aligned}
d_{n}= & -(0.22 \pm 0.03) e Q_{u} m_{u} \tilde{c}_{\gamma}^{(u)}+(0.74 \pm 0.07) e Q_{d} m_{d} \tilde{c}_{\gamma}^{(d)}+(0.0077 \pm 0.01) e Q_{s} m_{s} \tilde{c}_{\gamma}^{(s)} \\
& -(0.55 \pm 0.28) e m_{u} \tilde{c}_{g}^{(u)}-(1.1 \pm 0.55) e m_{d} \tilde{c}_{g}^{(d)} \pm(50 \pm 40) e g_{s} C_{\tilde{G}} \\
= & -\left\{(1.5 \pm 0.2)\left(v^{2} \tilde{c}_{\gamma}^{(u)}\right)+(5.2 \pm 0.5)\left(v^{2} \tilde{c}_{\gamma}^{(d)}\right)+(0.8 \pm 1.0)\left(v^{2} \tilde{c}_{\gamma}^{(s)}\right)\right. \\
& \left.+(5.5 \pm 2.8)\left(v^{2} \tilde{c}_{g}^{(u)}\right)+(23 \pm 12)\left(v^{2} \tilde{c}_{g}^{(d)}\right) \pm(345 \pm 276) v^{2} C_{\tilde{G}}\right\} \times 10^{-9} e \mathrm{fm},
\end{aligned}
$$

where all coefficients should be evaluated at $\mu=\Lambda_{\chi}$. Because of recent lattice calculations [47, 48], the contributions from the up- and down-quark EDMs in this expression are known to $O(15 \%)$, while 

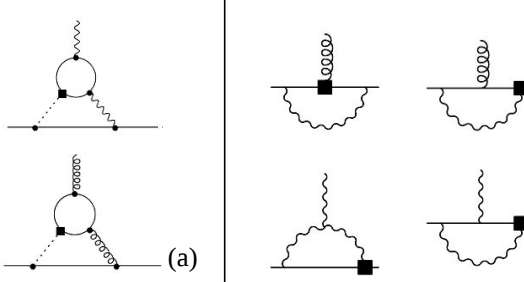

(b)
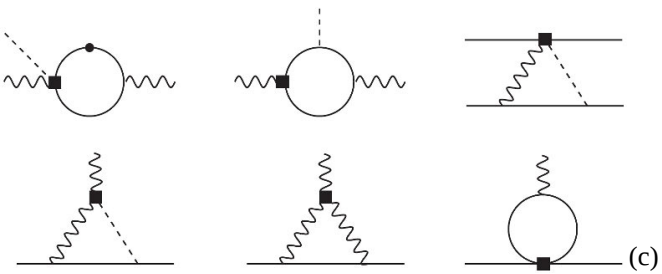

Figure 2. Representative diagrams of the matching of the top operators $C_{Y}, C_{g}, C_{\gamma}, C_{W b}$ and $C_{W t}$ onto the operators in Eq. (11). A solid line denotes a quark, a dashed line the Higgs boson. Wavy lines denote photons and EW bosons, while curly lines gluons. Squares denote the interactions in Eq. (1). (a) Barr-Zee diagrams representing the contribution of $C_{Y}$ to lepton and quark EDMs and MDMs, and quark CEDMs. (b) One-loop mixing of the top dipoles onto light quark EDMs and CEDMs. (c) Mixing of $C_{\gamma}$ into $\varphi^{2} X X$ and $L R L R$ fourfermion operators, and the mixing of the latter into light fermion electroweak dipoles.

the strange contribution is still highly uncertain. The quark CEDM contributions were calculated using QCD sum rules, and have an estimated 50\% uncertainty [42-45]. The situation is even more dire for the Weinberg operator, whose matrix element is known only at the order of magnitude level. Since the sign of this contribution is not well determined, we left it arbitrary and varied it in our analysis. Similar expressions can be derived for the ${ }^{199} \mathrm{Hg}$ EDM. In this case, in addition to the sizable hadronic uncertainties on the single nucleon EDM and the CP-violating pion-nucleon couplings, one has also to account for large errors in the nuclear structure calculations [51].

Having obtained the expressions for the observables in terms of the low-energy operators in Eq. (11), we now need to match the top operators in Eq. (1) onto $C_{\gamma}^{(l, q, b s)}, C_{g}^{(q, b s)}$ and $C_{\tilde{G}}$, and run down to the scale $\Lambda_{\chi}$. The anomalous Yukawa $C_{Y}$ is effectively a dimension-four coupling, and it does not mix onto the operators in Eq. (11) above the top threshold. Its contribution to the lepton and quark EDMs is induced by finite two-loop Barr-Zee diagrams [52], as represented in Fig. 2(a). The contribution of $C_{Y}$ to the flavor non-diagonal dipoles $C_{\gamma}^{(b s)}$ and $C_{g}^{(b s)}$ is further suppressed.

The dipole operators have a richer mixing structure $[6,11,53]$. Above the scale $m_{t}$, top dipoles mix with other dipoles. Representative diagrams are shown in Fig. 2(b). Since we start with heavy flavors, $t$ and $b$, and are interested in flavor diagonal dipole operators involving light flavors, and flavor changing dipoles involving a strange or down quark, these mixings in general involve $W$ loops, and some insertion of small CKM elements. This direct, one-loop mixing is the leading contribution to $B \rightarrow X_{s} \gamma$, giving

$$
\begin{aligned}
& C_{\gamma}^{(b s)}\left(m_{t}\right)=\left(6.4 C_{\gamma}(\Lambda)-0.12 C_{g}(\Lambda)+19 C_{W t}(\Lambda)-37 C_{W b}(\Lambda)\right) \cdot 10^{-4} \\
& C_{g}^{(b s)}\left(m_{t}\right)=-1.9 \cdot 10^{-4} C_{g}(\Lambda)
\end{aligned}
$$

where $\Lambda=1 \mathrm{TeV}$. Combining Eqs. (15) and (12), we see that $B \rightarrow X_{s} \gamma$ gives a strong constraint on the real part of $C_{W b},\left|v^{2} c_{W b}\right| \lesssim 0.05$. The imaginary part $\tilde{c}_{W b}$ is less constrained, $\left|v^{2} \tilde{c}_{W b}\right| \lesssim 0.3$. Similarly, one finds that $c_{W t}$ and $c_{\gamma}$ are constrained at the $10 \%$ and $30 \%$ level, and the constraints on the imaginary parts are weaker by a factor of 2-3.

Sizable corrections to the flavor diagonal EDM and MDM operators in Eq. (11) arise from a twostep mechanism, depicted in Fig. 2(c). First the top dipoles mix onto operators with two scalar fields and two gauge bosons, $\varphi^{2} X X$, or gauge-invariant four-fermion operators with $L R L R$ chiral structure, such as $\left(\bar{l}_{L} \sigma^{\mu \nu} e_{R}\right) \varepsilon_{I J} \bar{q}_{L}^{J} \sigma_{\mu \nu} u_{R}$. These operators then mix back onto lepton and light quark dipoles. The 
two-step mechanism induces contributions to the lepton and quark electric and magnetic moments that are suppressed by two loop factors, but are enhanced by a large double logarithm and do not contain small CKM elements. In several instances the two-step mechanism dominates the coefficients of the operators in Eq. (11).

As a concrete example, we can consider the mixing of the top EDM $\tilde{c}_{\gamma}$ onto the electron EDM. If one neglects the running of the top Yukawa coupling, the two-step path depicted in Fig. 2(c) leads to

$$
\frac{\tilde{c}_{\gamma}^{(e)}}{\tilde{c}_{\gamma}} \simeq \frac{3 N_{c} Q_{t} g^{4}}{(4 \pi)^{4}} \frac{m_{t}^{2}}{v^{2}}\left[1+\left(12 Q_{t}-1\right) t_{W}^{2}\right]\left(\log \frac{\Lambda}{m_{t}}\right)^{2} \sim 4 \cdot 10^{-4} .
$$

Together with Eq. (13), and the experimental limit on the electron EDM, Eq. (16) implies a very strong bound on $\tilde{c}_{\gamma},\left|v^{2} \tilde{c}_{\gamma}\right|<1.4 \cdot 10^{-3}$, much stronger than the bounds from $B \rightarrow X_{s} \gamma$ and collider.

The detailed solutions of the RGE equations are given in Ref. [6]. The main results are:

- the top EDM and weak EDM operators contribute mainly to the electron and the light quark EDMs, via the two-step mechanism described above. The loop factors cost a $10^{-4} \operatorname{suppression,} \tilde{c}_{\gamma}^{(e, q)}\left(\Lambda_{\chi}\right) \sim$ $10^{-4} \tilde{c}_{\gamma, W t}(\Lambda)$

- the top CEDM gives a large threshold contribution to the Weinberg operator, $C_{\tilde{G}}$, and it contributes to the light quark CEDM via the two-step mechanism, The loop suppression is at the $10^{-3}$ level, $C_{\tilde{G}}\left(\Lambda_{\chi}\right), \tilde{c}_{g}^{(q)}\left(\Lambda_{\chi}\right) \sim 10^{-3} \tilde{c}_{g}(\Lambda)$. The top CEDM contributes to $d_{e}$ at the $10^{-5}$ level,

- the bottom quark EDM operator $\tilde{c}_{W b}$ predominantly generates the Weinberg operator, via threshold corrections at the $b$ scale, and the light quark CEDMs. The contributions are at the $10^{-5}$ level,

- the top pseudoscalar Yukawa coupling $\tilde{c}_{Y}$ gives threshold corrections to the electron and quark EDMs and to the quark CEDM, at the $10^{-4}-10^{-5}$ level.

From Eqs. (13) and (14), one can see that the electron EDM constrains $\tilde{c}_{\gamma}$ and $\tilde{c}_{W t}$ at the permil level. These bounds are not significantly affected by theory uncertainties. The Yukawa coupling $\tilde{c}_{Y}$ is also mainly constrained by the electron EDM, but the bound is roughly a factor of 10 weaker. In light of the large contribution to the Weinberg operator, the top CEDM is naively constrained by the neutron EDM at the $10^{-4}$ level. $\tilde{c}_{W b}$ is also strongly constrained by the neutron EDM. In these cases, however, one has to take into account the large nonperturbative uncertainties. In our analysis, we deal with the theoretical uncertainties in two different ways. In what we call "central" strategy we neglect theoretical uncertainties in the hadronic and nuclear matrix elements entering $d_{n}, d_{\mathrm{Hg}}$ and $A_{C P}$. While excessively optimistic, this strategy allows to appreciate the full potential of EDM experiments. In the "R-fit" strategy we vary all theoretical uncertainties within the allowed ranges assuming a flat distribution, and minimize the total $\chi^{2}$. This method corresponds to the Range-fit (R-fit) procedure defined in Ref. [54]. With the current theoretical knowledge, the bounds obtained with these two strategies are very different, highlighting the importance of improving the determination of hadronic (and nuclear) matrix elements for EDMs.

The same mechanism that induces the electron EDM also generates magnetic dipole moments of charged leptons, proportional to the real part of the couplings in Eq. (1). In Ref. [6] we showed that $\left\{v^{2} c_{\gamma}, v^{2} c_{W t}\right\} \simeq O(10)$ and $\left\{v^{2} c_{g}, v^{2} c_{Y}\right\} \simeq O(100)$ are needed to ease the tension between theory and experiment in the muon $g-2$. Such large values are already excluded by collider constraints, so that the $g-2$ anomaly cannot be due to the dimension-six operators we investigate.

\section{Combined bounds on top and Higgs couplings}

In this Section we discuss the combined collider and low energy bounds on the operators introduced in Eq. (1). We start with a single coupling analysis, in which we set only one of the complex coefficients 

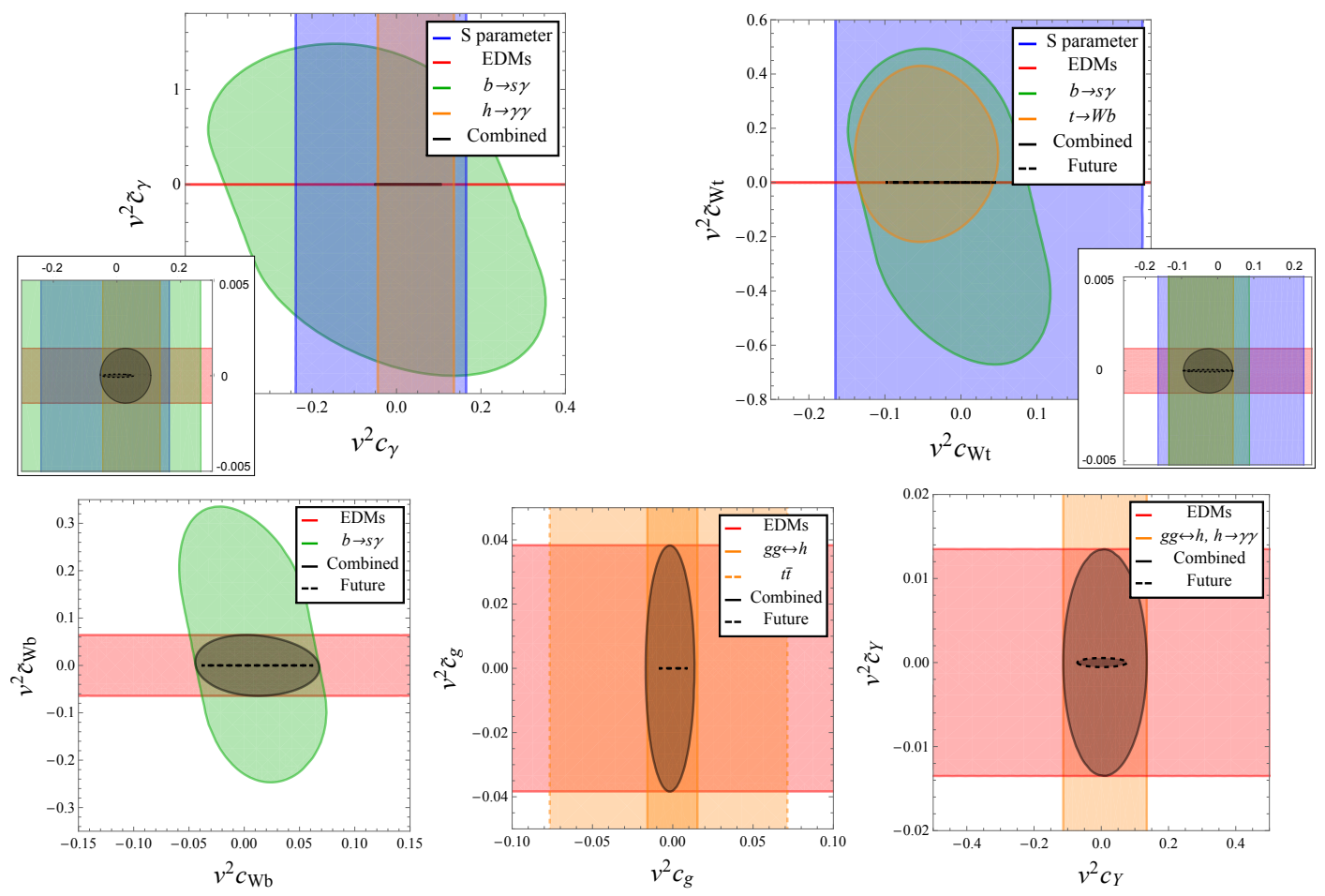

Figure 3. $90 \%$ C.L. allowed regions in the $v^{2} c_{\gamma}-v^{2} \tilde{c}_{\gamma}$ (top left panel), $v^{2} c_{W t}-v^{2} \tilde{c}_{W t}$ (top right panel), $v^{2} c_{W b}-v^{2} \tilde{c}_{W b}$ (bottom left panel) and $v^{2} c_{g}-v^{2} \tilde{c}_{g}$ (bottom center panel) and $v^{2} c_{Y}-v^{2} \tilde{c}_{Y}$ (bottom right panel) planes, with couplings evaluated at $\Lambda=1 \mathrm{TeV}$.

in Eq. (1) to be different from zero at the scale $\Lambda=1 \mathrm{TeV}$. The $90 \%$ C.L. allowed region for $C_{\gamma}, C_{W t}$, $C_{W b}, C_{g}$ and $C_{Y}$, obtained with the R-fit strategy, is shown in Fig. 3. In black we show the combined allowed region, while the other regions denote the most constraining bounds coming from EDMs (red), $B \rightarrow X_{s} \gamma$ (green), electroweak precision observables (blue), and direct and indirect collider searches (orange). The dashed black contour denotes future sensitivities, considering improvements in EDM experiments [55], super-B factory measurements [56, 57], and collider searches [28, 29].

In the single operator analysis, EDMs very effectively constrain the imaginary parts of the coefficients $C_{\alpha}$. This is true in particular for $\tilde{c}_{\gamma}$ and $\tilde{c}_{W t}$, which are constrained at the permil level by the electron EDM. As Fig. 3 clearly illustrates, the EDM bounds are a factor of $10^{3}$ stronger than flavor and collider observables [5,6]. The real part of the couplings, $c_{\gamma}$ and $c_{W t}$, can be larger. $c_{\gamma}$ is mainly constrained by Higgs decay into photons. We find the allowed region for $c_{\gamma}$ to be $-0.05<v^{2} c_{\gamma}<0.11$. $c_{W t}$ receives competitive constraints from $B \rightarrow X_{s} \gamma$, the $S$ parameter, single top production, and the $W$ boson helicity fractions. The combined allowed region for $c_{W t}$ is $-0.10<v^{2} c_{W t}<0.04$. In the single coupling analysis, the bounds on $C_{\gamma}$ and $C_{W t}$ are equal in the central and R-fit strategies.

The imaginary part of $C_{W b}$ is also well constrained by EDMs, in particular the neutron, giving $\left|v^{2} \tilde{c}_{W b}\right|<0.06$. The EDM bound is five time stronger than the limit extracted from $A_{C P}$, even with the conservative treatment of the theoretical errors that we are adopting. In this case, going from the 
central to the more conservative R-fit strategy costs almost one order of magnitude. The bound on $c_{W b}$ is determined by the $B \rightarrow X_{s} \gamma$ branching ratio, yielding $-0.04<v^{2} c_{W b}<0.07$.

As we discussed earlier, $\tilde{c}_{g}$ contributes to the neutron and electron EDMs. The induced neutron EDM is about 100 times larger than $d_{e}$, so that naively one would expect the strongest constraints to come from $d_{n}$. In the R-fit procedure, however, $d_{n}$ is subject to cancellations between the contributions of the Weinberg operator and of the light quark (C)EDMs, and the cancellations are so severe that the best limit actually comes from $d_{e}$, which gives $\left|v^{2} \tilde{c}_{g}\right|<0.038$. In the central strategy, where there is no room for cancellations, the neutron EDM is indeed dominant, giving a bound $\left|v^{2} \tilde{c}_{g}\right|<2.9 \cdot 10^{-4}$. This dramatic effect illustrates the fact that, to better constrain top couplings, it is crucial to get the matrix elements of the Weinberg operator under control. The real part of the coupling, $c_{g}$, is strongly constrained by $g g \rightarrow h$, which gives $-0.017<v^{2} c_{g}<0.014$. At the moment, direct observables such as $t \bar{t}$ and $t \bar{t} h$ production give weaker bounds, but it will be very interesting to study the evolution of these constraints at the LHC Run II.

Finally, the pseudoscalar Yukawa, $\tilde{c}_{Y}$, receives its strongest bound from the electron EDM, $\left|v^{2} \tilde{c}_{Y}\right|<$ 0.013. The real Yukawa, $c_{Y}$, affects the Higgs gluon fusion production cross section and the decays $h \rightarrow g g$ and $h \rightarrow \gamma \gamma . c_{Y}$ can also be probed directly by studying the associated $t \bar{t} h$ production. With the current experimental data, the bound is dominated by the Higgs signal strengths and is at the $10 \%$ level, $-0.12<v^{2} c_{Y}<0.14$. The bound from $t \bar{t} h$ is noticeably weaker.

The bounds on the real and imaginary parts of $C_{\gamma}, C_{W t}, C_{W b}, C_{g}$ and $C_{Y}$ are weakened by allowing all coefficients to be non-zero at the scale $\Lambda$. This is particularly true for the imaginary parts, for which one does not have as many sensitive observables as for the real couplings. Still, in order to evade the strong EDM bounds obtained in the single coupling scenario, the couplings need to exhibit strong correlations, which provide non trivial constraints on new physics models.

In conclusion, we have studied high and low energy constraints on chirality-flipping top-Higgs couplings, including a complex anomalous Yukawa coupling, and four dipole interactions. We have found that the real parts of the couplings are bound by a combination of direct and indirect probes. In particular, Higgs production and decay are very sensitive probes, competitive with, or even more sensitive than, processes with top final states. The imaginary part of the couplings are very well constrained by EDMs, in particular by the electron EDM $d_{e}$. To improve the bounds from nuclear and hadronic EDMs, it is crucial to reduce the theoretical uncertainties on nonperturbative matrix elements.

\section{References}

[1] G. Aad et al. (ATLAS Collaboration), Phys.Lett. B716, 1 (2012), 1207. 7214

[2] S. Chatrchyan et al. (CMS Collaboration), Phys.Lett. B716, 30 (2012), 1207.7235

[3] G. Aad et al. (ATLAS, CMS), JHEP 08, 045 (2016), 1606.02266

[4] Y.T. Chien, V. Cirigliano, W. Dekens, J. de Vries, E. Mereghetti, JHEP 02, 011 (2016), 1510.00725

[5] V. Cirigliano, W. Dekens, J. de Vries, E. Mereghetti, Phys. Rev. D94, 016002 (2016), 1603.03049

[6] V. Cirigliano, W. Dekens, J. de Vries, E. Mereghetti, Phys. Rev. D94, 034031 (2016), 1605.04311

[7] W. Buchmüller, D. Wyler, Nucl. Phys. B 268, 621 (1986)

[8] B. Grzadkowski, M. Iskrzynski, M. Misiak, J. Rosiek, JHEP 1010, 085 (2010), 1008 . 4884

[9] E.E. Jenkins, A.V. Manohar, M. Trott, JHEP 10, 087 (2013), 1308. 2627

[10] E.E. Jenkins, A.V. Manohar, M. Trott, JHEP 01, 035 (2014), 1310. 4838 
[11] R. Alonso, E.E. Jenkins, A.V. Manohar, M. Trott, JHEP 04, 159 (2014), 1312 . 2014

[12] D. Atwood, A. Kagan, T.G. Rizzo, Phys. Rev. D52, 6264 (1995), hep-ph/9407408

[13] P. Haberl, O. Nachtmann, A. Wilch, Phys. Rev. D53, 4875 (1996), hep-ph/9505409

[14] M. Czakon, A. Mitov, Comput. Phys. Commun. 185, 2930 (2014), 1112 . 5675

[15] M. Czakon, P. Fiedler, A. Mitov, Phys. Rev. Lett. 110, 252004 (2013), 1303.6254

[16] S. Heinemeyer et al. (LHC Higgs Cross Section Working Group) (2013), 1307 . 1347

[17] G. Aad et al. (ATLAS), Eur. Phys. J. C76, 6 (2016), 1507.04548

[18] V. Khachatryan et al. (CMS), Eur. Phys. J. C75, 212 (2015), 1412. 8662

[19] T.A. Aaltonen et al. (CDF, D0), Phys. Rev. D89, 072001 (2014), 1309.7570

[20] G. Aad et al. (ATLAS), Eur. Phys. J. C74, 3109 (2014), 1406. 5375

[21] S. Chatrchyan et al. (CMS), JHEP 02, 024 (2014), [Erratum: JHEP02,102(2014)], 1312.7582

[22] O. Bessidskaia Bylund, F. Maltoni, I. Tsinikos, E. Vryonidou, C. Zhang, JHEP 05, 052 (2016), 1601.08193

[23] M. Schulze, Y. Soreq, Eur. Phys. J. C76, 466 (2016), 1603. 08911

[24] J. Boudreau, C. Escobar, J. Mueller, K. Sapp, J. Su (2013), 1304. 5639

[25] G. Aad et al. (ATLAS), JHEP 04, 023 (2016), 1510.03764

[26] A. Czarnecki, J.G. Korner, J.H. Piclum, Phys. Rev. D81, 111503 (2010), 1005.2625

[27] J. Drobnak, S. Fajfer, J.F. Kamenik, Phys. Rev. D82, 114008 (2010), 1010. 2402

[28] Projected Performance of an Upgraded CMS Detector at the LHC and HL-LHC: Contribution to the Snowmass Process, in Community Summer Study 2013: Snowmass on the Mississippi (CSS2013) Minneapolis, MN, USA, July 29-August 6, 2013 (2013), 1307.7135, http://inspirehep.net/record/1244669/files/arXiv: 1307.7135.pdf

[29] Tech. Rep. ATL-PHYS-PUB-2014-016, CERN, Geneva (2014), http : //cds . cern. ch/record/1956710

[30] K.A. Olive et al. (Particle Data Group), Chin. Phys. C38, 090001 (2014)

[31] Y. Amhis et al. (Heavy Flavor Averaging Group (HFAG)) (2014), 1412.7515

[32] J. Baron et al. (ACME Collaboration), Science 343, 269 (2014), 1310. 7534

[33] C.A. Baker, D.D. Doyle, P. Geltenbort, K. Green, M.G.D. van der Grinten et al., Phys. Rev. Lett. 97, 131801 (2006), hep-ex/0602020

[34] J. Pendlebury et al., Phys. Rev. D92, 092003 (2015), 1509.04411

[35] B. Graner, Y. Chen, E.G. Lindahl, B.R. Heckel, Phys. Rev. Lett. 116, 161601 (2016), 1601.04339

[36] A.L. Kagan, M. Neubert, Eur. Phys. J. C7, 5 (1999), hep-ph/9805303

[37] E. Lunghi, J. Matias, JHEP 04, 058 (2007), hep-ph/0612166

[38] M. Misiak et al., Phys. Rev. Lett. 98, 022002 (2007), hep-ph/0609232

[39] M. Misiak et al., Phys. Rev. Lett. 114, 221801 (2015), 1503.01789

[40] M. Czakon, P. Fiedler, T. Huber, M. Misiak, T. Schutzmeier, M. Steinhauser, JHEP 04, 168 (2015), 1503.01791

[41] M. Benzke, S.J. Lee, M. Neubert, G. Paz, Phys. Rev. Lett. 106, 141801 (2011), 1012 . 3167

[42] M. Pospelov, A. Ritz, Phys. Rev. D 63, 073015 (2001), hep-ph/0010037

[43] O. Lebedev, K.A. Olive, M. Pospelov, A. Ritz, Phys. Rev. D 70, 016003 (2004), hep-ph/0402023

[44] M. Pospelov, A. Ritz, Annals Phys. 318, 119 (2005), hep-ph/0504231

[45] J. Hisano, J.Y. Lee, N. Nagata, Y. Shimizu, Phys. Rev. D 85, 114044 (2012), 1204.2653 
[46] D.A. Demir, M. Pospelov, A. Ritz, Phys. Rev. D 67, 015007 (2003), hep-ph/0208257

[47] T. Bhattacharya, V. Cirigliano, R. Gupta, H.W. Lin, B. Yoon, Phys. Rev. Lett. 115, 212002 (2015), 1506.04196

[48] T. Bhattacharya, V. Cirigliano, S. Cohen, R. Gupta, A. Joseph, H.W. Lin, B. Yoon (PNDME), Phys. Rev. D92, 094511 (2015), 1506.06411

[49] J. de Vries, E. Mereghetti, R.G.E. Timmermans, U. van Kolck, Annals Phys. 338, 50 (2013), 1212.0990

[50] J. Bsaisou, U.G. Meißner, A. Nogga, A. Wirzba, Annals Phys. 359, 317 (2015), 1412 . 5471

[51] J. Engel, M.J. Ramsey-Musolf, U. van Kolck, Prog. Part. Nucl. Phys. 71, 21 (2013), 1303. 2371

[52] S.M. Barr, A. Zee, Phys. Rev. Lett. 65, 21 (1990)

[53] W. Dekens, J. de Vries, JHEP 1305, 149 (2013), 1303.3156

[54] J. Charles, A. Hocker, H. Lacker, S. Laplace, F.R. Le Diberder, J. Malcles, J. Ocariz, M. Pivk, L. Roos (CKMfitter Group), Eur. Phys. J. C41, 1 (2005), hep-ph/0406184

[55] K. Kumar, Z.T. Lu, M.J. Ramsey-Musolf, Working Group Report: Nucleons, Nuclei, and Atoms, in Community Summer Study 2013: Snowmass on the Mississippi (CSS2013) Minneapolis, MN, USA, July 29-August 6, 2013 (2013), 1312.5416, http://inspirehep.net/record/1272872/files/arXiv: 1312.5416.pdf

[56] M. Bona et al. (SuperB) (2007), 0709.0451

[57] S. Nishida, Experimental Prospects for $B \rightarrow X_{s / d} \gamma$ and $B->X_{s} \ell^{+} \ell^{-}$, in $C K M$ unitarity triangle. Proceedings, 6th International Workshop, CKM 2010, Warwick, UK, September 6-10, 2010 (2011), 1102.1045, http://inspirehep.net/record/888811/files/arXiv: 1102.1045.pdf 